\title{
Methotrexate-associated Lymphoproliferative Disorder in the Liver Resembling Hepatocellular Carcinoma Treated with Transarterial Chemoembolization
}

\author{
Mio Tsuruoka, Jun Inoue, Eiji Kakazu, Masashi Ninomiya, Tomoaki Iwata, Akitoshi Sano and \\ Atsushi Masamune
}

\begin{abstract}
:
Methotrexate-related lymphoproliferative disorder (MTX-LPD) is known to be a side effect of MTX, but its involvement in the liver has been rarely reported. We herein report a 70-year-old woman with autoimmune hepatitis and rheumatoid arthritis who developed multiple liver tumors. We initially considered that she had developed rapid-growing hepatocellular carcinoma (HCC) in the cirrhotic liver based on imaging tests. A tumor biopsy and transcatheter arterial chemoembolization were thus performed. The tumors were then diagnosed as diffuse large B-cell lymphoma pathologically and considered to be MTX-LPD. This case indicates that MTX-LPD should be considered even in cirrhotic patients with liver tumors resembling HCC.
\end{abstract}

Key words: MTX-LPD, AIH, RA, liver tumor, DLBCL

(Intern Med 59: 2255-2260, 2020)

(DOI: 10.2169/internalmedicine.4787-20)

\section{Introduction}

Methotrexate (MTX) is a folate antagonist that is recognized as an anchor drug for rheumatoid arthritis (RA) worldwide. Because of several adverse effects, such as bonemarrow disorder, pneumonia, infectious diseases, gastrointestinal tract disturbances, and liver dysfunction, including reactivation of hepatitis $\mathrm{B}$ virus, physicians should pay close attention to the patient's condition at all times during the treatment (1).

Lymphoproliferative disorder (LPD), including malignant lymphoma, is a rare but life-threatening complication in patients with RA $(2,3)$. The risk of LPD is higher in RA patients than in the general population $(4,5)$, and based on reports on the regression of LPD after MTX cessation (6), LPD has been considered to occur during the suppression of immune surveillance with MTX administration (3). LPD that developed under the administration of immunosuppressive agents was categorized as 'other-iatrogenic immunodeficiency-associated LPD' in the 2008 World Health Organization classification of tumors or hematopoie- tic and lymphoid tissues (7). MTX-associated LPD (MTXLPD) is found not only in lymph nodes but also several other organs such as the intestinal tract, skin, and lung (8), but its involvement in the liver is very rare, and the etiology and clinical outcome are largely unknown (9). In addition, the typical imaging features of MTX-LPD in the liver have not been defined, and its diagnosis by imaging tests is considered difficult $(10,11)$.

We herein report a case with MTX-LPD in the liver of a patient with autoimmune hepatitis (AIH) who had been treated for RA with MTX for as long as 17 years. Because the imaging findings of the liver tumors resembled hepatocellular carcinoma (HCC) and they grew rapidly, we performed transcatheter arterial chemoembolization (TACE) for the tumors before the pathological diagnosis had been made.

\section{Case Report}

A 70-year-old woman was found to have multiple liver tumors on computed tomography (CT) at a regular visit for the follow-up of AIH. Regarding her medical history, she had been diagnosed with $\mathrm{AIH}$ at 27 years old and RA at 38

Division of Gastroenterology, Tohoku University Graduate School of Medicine, Japan

Received: March 4, 2020; Accepted: April 21, 2020; Advance Publication by J-STAGE: June 9, 2020

Correspondence to Dr. Jun Inoue, jinoue-drgn@umin.net 

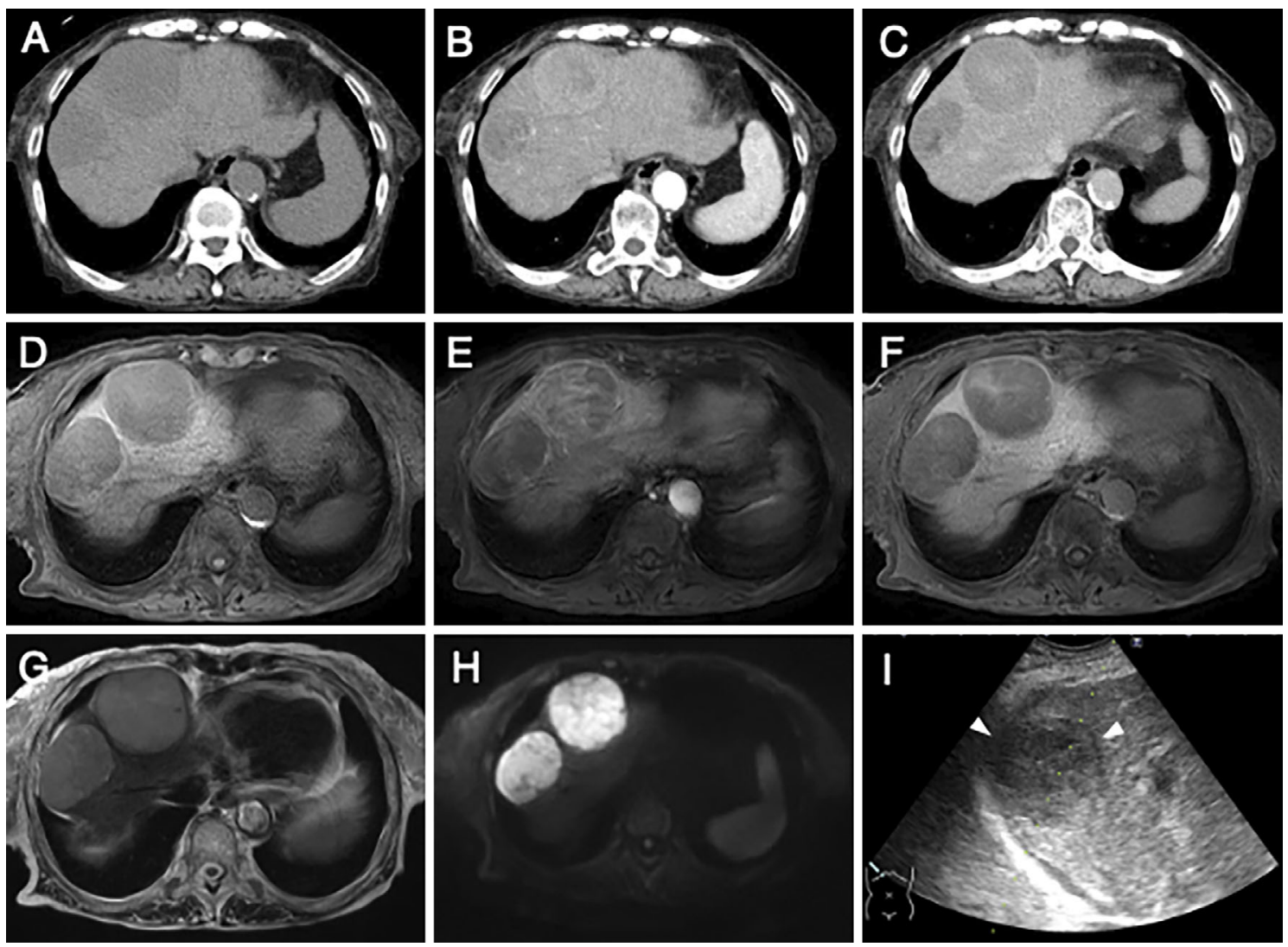

Figure 1. Images of abdominal contrast-enhanced computed tomography (CT), magnetic resonance imaging (MRI) and ultrasonography (US) in the present case. The multiple liver tumors showed a low density in the precontrast phase (A), heterogenous enhancement in the early phase (B) and wash-out with corona-like enhancement in the late phase (C). The tumors exhibited hypointensity on precontrast T1-weighted MRI images (D), slight early enhancement with gadolinium-ethoxybenzyl diethylenetriamine-pentaacetic acid (Gd-EOB-DTPA) in the early phase (E) and hypointensity in the hepatobiliary phase (F). They exhibited slight hyperintensity on T2-weighted MRI images (G) and hyperintensity on diffusion-weighted images $(\mathrm{H})$. Ultrasound sonography showed low-echoic tumors (arrowheads) in the same area as on CT and MRI (I).

years old, and the administration of MTX and salazosulfapyridine (SASP) had been started as RA treatment 17 years ago. Subsequently, prednisolone had been started and maintained at $5 \mathrm{mg}$ per day. In addition, she had a history of appendicitis at 17 years old and had been diagnosed with hypothyroidism around 40 years old. She underwent knee operations for RA at 52 and 63 years old. She had no familial history of liver diseases or collagen diseases.

At a regular visit in September 2018, ultrasonography (US) for the abdomen showed that the internal liver was rough, and the liver surface was irregular. We considered her to have liver cirrhosis due to AIH. At that time, no liver tumors were noted. Four months later (January 2019), contrast-enhanced CT showed multiple liver tumors with a maximum diameter of $55 \mathrm{~mm}$ (Fig. 1A-C). The main lesions were found in $\mathrm{S} 4 / 8$ and $\mathrm{S} 3$, and additional small lesions were found in S4 and S8. They exhibited heterogeneous enhancement at the early phase and wash-out at the late phase as well as corona-like enhancement surrounding the tumors. Contrast-enhanced magnetic resonance imaging (MRI) with gadolinium-ethoxybenzyl diethylenetriamine-pentaacetic acid
(Gd-EOB-DTPA) showed a similar enhancement pattern in the tumors and low intensity at the hepatobiliary phase (Fig. 1D-E).

The clinical parameters are shown in Table. The patient had a low serum albumin level and low platelet count, findings that were consistent with liver cirrhosis. We considered the liver tumors to be compatible with HCC based on the presence of liver cirrhosis and the typical CT/MRI findings. Although serum tumor markers, such as alpha-fetoprotein (AFP) and protein induced by vitamin $\mathrm{K}$ absence or antagonist-II (PIVKA-II), were within the normal range, we considered them not to be essential for the diagnosis of HCC $(12,13)$. Because of the rapid growth of the tumors, we performed TACE soon after the US-guided tumor biopsy. TACE was selected based on the guidelines of HCC treatment by American Association for the Study of Liver Diseases (AASLD) (14) and the Japan Society of Hepatology (JSH) (15). The digital subtraction angiography (DSA) and CT images obtained during TACE are shown in Fig. 2. DSA showed that the main feeder of the hepatic segment 4/8 (S4/ 8) tumor was the middle hepatic artery (Fig. 2A). CT during 
Table. Laboratory Findings on Admission.

\begin{tabular}{lclclc}
\hline WBC & $4,200 / \mu \mathrm{L}$ & TP & $6.0 \mathrm{~g} / \mathrm{dL}$ & IgG & $1,559 \mathrm{mg} / \mathrm{dL}$ \\
Neut & $61.1 \%$ & Albumin & $2.9 \mathrm{~g} / \mathrm{dL}$ & IgA & $175 \mathrm{mg} / \mathrm{dL}$ \\
Eosi & $1.7 \%$ & T-Bil & $0.5 \mathrm{mg} / \mathrm{dL}$ & IgM & $145 \mathrm{mg} / \mathrm{dL}$ \\
Baso & $0.2 \%$ & ALP & $327 \mathrm{U} / \mathrm{L}$ & ANA & $\times 80$ \\
Mono & $5.7 \%$ & $\gamma$-GTP & $111 \mathrm{U} / \mathrm{L}$ & AFP & $7.8 \mathrm{ng} / \mathrm{mL}$ \\
Lymp & $31.3 \%$ & AST & $63 \mathrm{U} / \mathrm{L}$ & AFP-L3 & $<0.5 \%$ \\
RBC & $2.69 \times 10^{6} / \mu \mathrm{L}$ & ALT & $34 \mathrm{U} / \mathrm{L}$ & PIVKA-II & $38 \mathrm{mAU} / \mathrm{mL}$ \\
MCV & $109.7 \mathrm{fL}$ & LDH & $241 \mathrm{U} / \mathrm{L}$ & HBsAg & $(-)$ \\
MCH & $35.7 \mathrm{pg}$ & BUN & $21 \mathrm{mg} / \mathrm{dL}$ & Anti-HCV & $(-)$ \\
MCHC & $32.5 \mathrm{~g} / \mathrm{dL}$ & Creatinine & $0.65 \mathrm{mg} / \mathrm{dL}$ & & \\
Hb & $9.6 \mathrm{~g} / \mathrm{dL}$ & Ureic acid & $5.9 \mathrm{mg} / \mathrm{dL}$ & & \\
Hct & $29.5 \%$ & Na & $145 \mathrm{mEq} / \mathrm{L}$ & & \\
PLT & $91 \times 10^{3} / \mu \mathrm{L}$ & $\mathrm{K}$ & $4.1 \mathrm{mEq} / \mathrm{L}$ & & \\
PT & $92.3 \%$ & $\mathrm{Cl}$ & $111 \mathrm{mEq} / \mathrm{L}$ & & \\
APTT & $28.9 \mathrm{sec}$ & $\mathrm{Ca}$ & $8.3 \mathrm{mg} / \mathrm{dL}$ & & \\
\hline
\end{tabular}

WBC: white blood cells, Neut: neutrophils, Eosi: eosinophils, Baso: basophils, Mono: monocytes, Lymp: lymphocytes, RBC: red blood cells, MCV: mean corpuscular volume, $\mathrm{MCH}$ : mean corpuscular hemoglobin, MCHC: mean corpuscular hemoglobin concentration, Hb: hemoglobin, Hct: hematocrit, PLT: platelet, PT: prothrombin time, APTT: activated partial thromboplastin time, TP: total protein, T-Bil: total bilirubin, ALP: alkaline phosphatase, $\gamma$-GTP: $\gamma$-glutamyltransferase, AST: aspartate aminotransferase, ALT: alanine aminotransferase, LDH: lactate dehydrogenase, BUN: blood urea nitrogen, Ig: immunoglobulin, ANA: anti-nuclear antibody, AFP: alpha-fetoprotein, PIVKA-II: protein induced by vitamin $\mathrm{K}$ absence or antagonist-II, HBsAg: hepatitis B surface antigen, anti-HCV: antibody against hepatitis $\mathrm{C}$ virus

arterial portography (CTAP) via the superior mesenteric artery showed enhancement defects in S4/8 and S3 of the liver (Fig. 2B), and CT arteriography (CTA) showed clear enhancement in the same area (Fig. 2C, D). The middle hepatic artery feeding the S4/8 tumor and hepatic artery 4 (A 4) feeding the $\mathrm{S} 3$ tumor were then embolized with drugeluting beads (DC bead ${ }^{\circledR}$; Biocompatibles, London, UK) that had been loaded with epirubicin and gelatin sponge particles.

One week after TACE, CT showed shrinkage of the tumors with a maximum diameter of $30 \mathrm{~mm}$ and the appearance of a small volume of ascites (Fig. 3A), so TACE was considered to have been effective for the tumors. The patient was discharged 10 days after TACE, and the pathological diagnosis of the tumors was subsequently made (Fig. 4). Atypical cells with densely stained nuclei that resembled a stripped nucleus were found to be growing solidly. The shape of the nuclei was irregular, and division of the nuclei was observed (Fig. 4A, B). Immunostaining revealed the characteristics of the tumors as follows (Fig. 4C, D): AE1/ AE3 (-), aSMA (-), bcl2 (+), bcl6 ( \pm ), CD3 (-), CD5 (-), CD10 (-), CD20 (+), CD23 ( \pm ), CD34 (-), CD38 (-), CD 45 (+), CD79a (+), CD138 (-), CK7 (-), cyclinD1 (-),

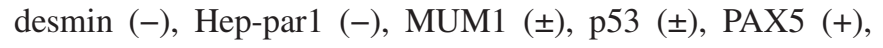
S-100 (-), vimentin $(+), \kappa$ light chain $(-), \lambda$ light chain $(-)$, and Ki-67 90\%. DNA of Epstein-Barr virus (EBV) was detected in the cells with an in situ hybridization method. Based on these results, the tumors were diagnosed as diffuse large B-cell lymphoma (DLBCL), and we consulted a hematologist in our hospital.

We then suspected that the liver tumors might be MTX-
LPD, and MTX was discontinued three weeks after the TACE. At this point, the soluble IL-2 receptor level was 793 $\mathrm{U} / \mathrm{mL}$. Regarding the serum EBV markers, the anti-viral capsid antigen IgG, EBV-nuclear antigen antibody and EBV DNA values were 2560 (cut-off: 0.5), 160 (cut-off: 0.5) and 670 copies/mL, respectively. CT performed four weeks after TACE showed the further shrinkage of the liver tumors (Fig. 3B). Positron emission tomography-CT (PET-CT) at two and five months after TACE showed no abnormal accumulation of ${ }^{18} \mathrm{~F}$-fluorodeoxyglucose. CT performed 10 months after TACE showed only small scars in the same area (Fig. 3C). Therefore, we considered the liver tumor of MTX-LPD to have regressed completely.

\section{Discussion}

MTX-LPD was reported first by Ellman et al. in 1991 (16). Although the mechanism of occurrence remains unclear, histology has shown that it is derived from B cells mainly, and an association with EBV was suggested $(17,18)$. Although a high dose of MTX was reported to be a risk factor of MTX-LPD (19), this remains controversial. The mean duration of MTX administration before MTX-LPD development was reported to be 30 months, and the duration of MTX administration is not considered to be associated with MTX-LPD development (20, 21). Pathologically, DLBCL is the type most frequently found and accounts for 35-60\% of MTX-LPD cases, followed by Hodgkin lymphoma, which accounts for $12-25 \%$ of cases (17).

No diagnostic criteria of MTX-LPD have yet been established. In Japan, the Japan College of Rheumatology recom- 

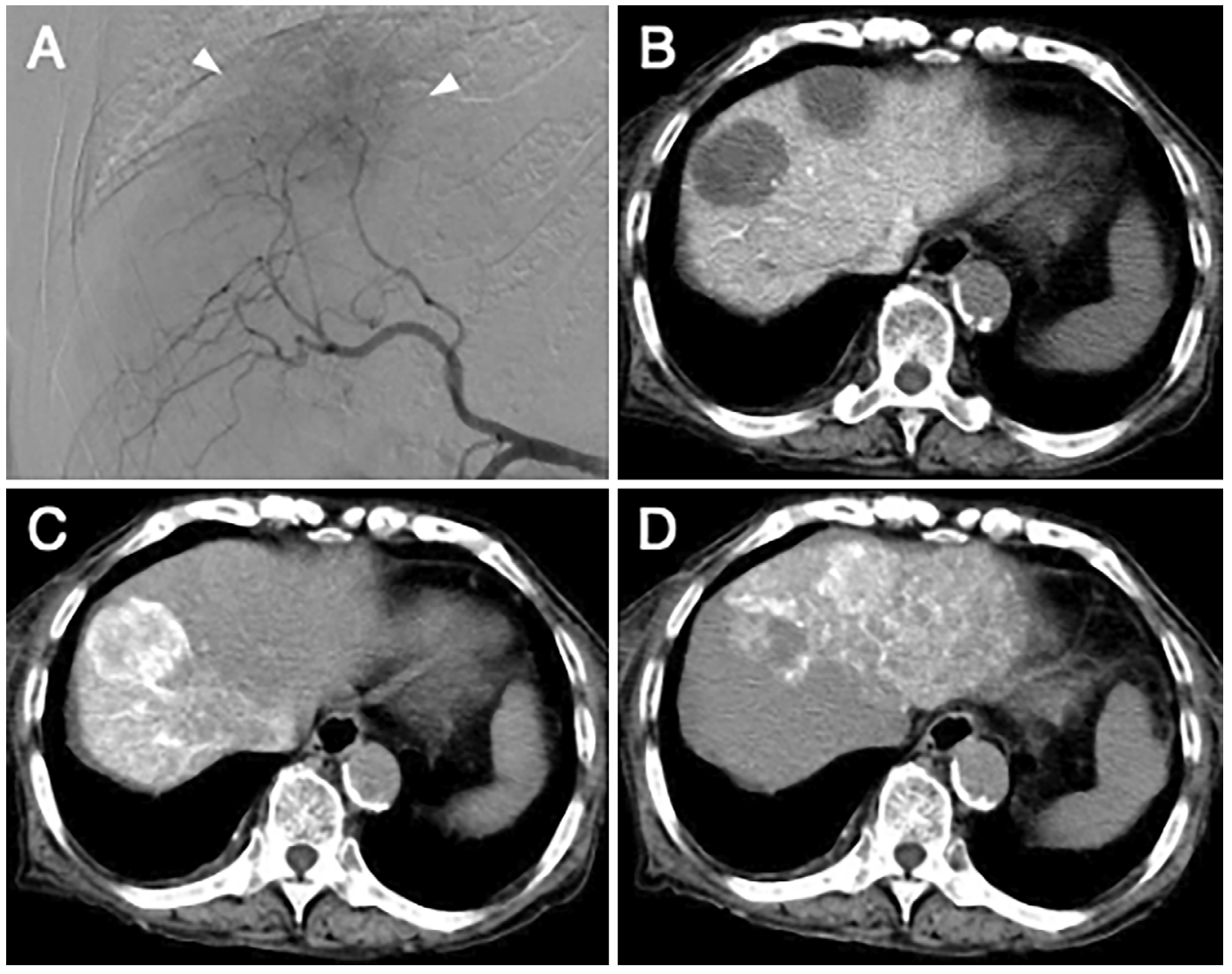

Figure 2. Images during transcatheter arterial chemoembolization (TACE). (A) Digital subtraction angiography via the common hepatic artery. The middle hepatic artery and right hepatic artery branched off from proper hepatic artery. Separately, the right hepatic artery branched off from the left gastric artery (not shown). The arrowhead indicates weak staining in the tumors that were fed mainly by the middle hepatic artery. (B) CT during arterial portography (CTAP) showed perfusion defects mainly in S4/8 and S3 of the liver. CT angiography confirmed that the S4/8 tumor was fed by the middle hepatic artery (C), while the $S 3$ tumor was fed by the left hepatic artery (D). TACE was performed from these arteries.
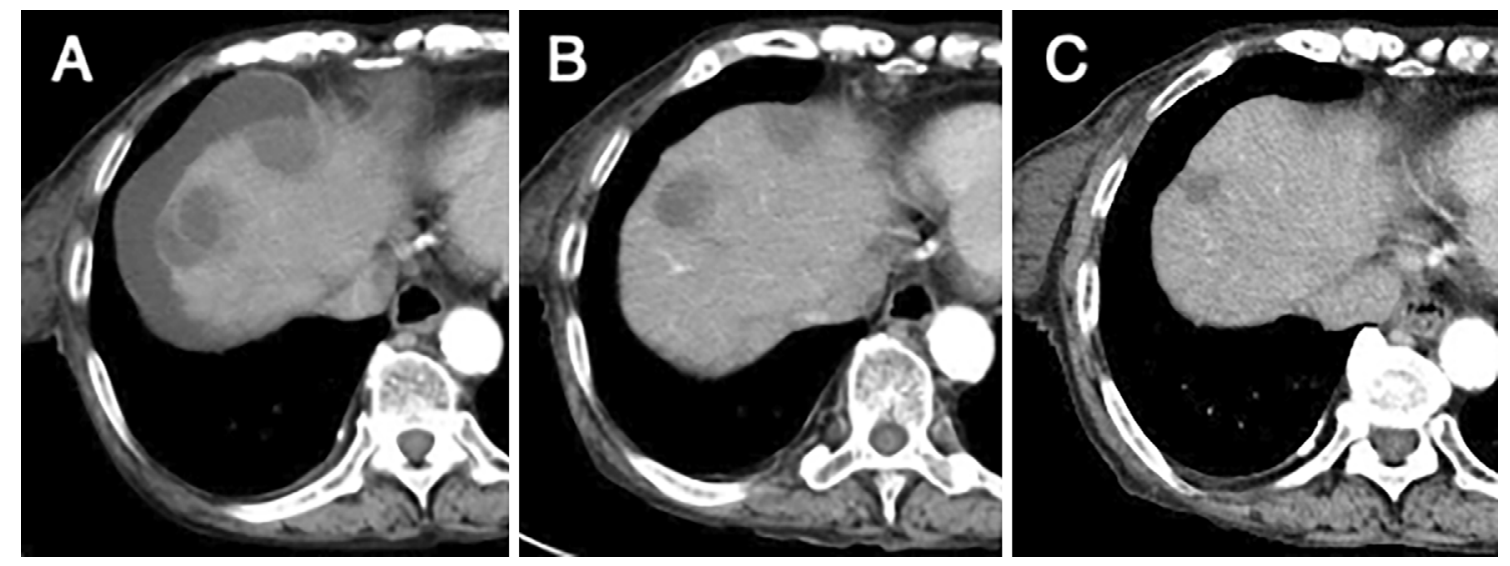

Figure 3. CT images after TACE and cessation of MTX. The liver tumors shrank serially 1 week (A), 4 weeks (B) and 10 months after TACE (C).

mends considering the possibility of MTX-LPD if RA patients who are being treated with MTX present with systemic symptoms, such as an undefined fever, general malaise and body weight loss, or show abnormal test results, such as hepatosplenomegaly, abnormal differential count of leukocytes, a decreased platelet count, anemia and high lactic acid dehydrogenase (1). Patients with lymph node swelling or extranodal diseases should be cared for as well (22). 


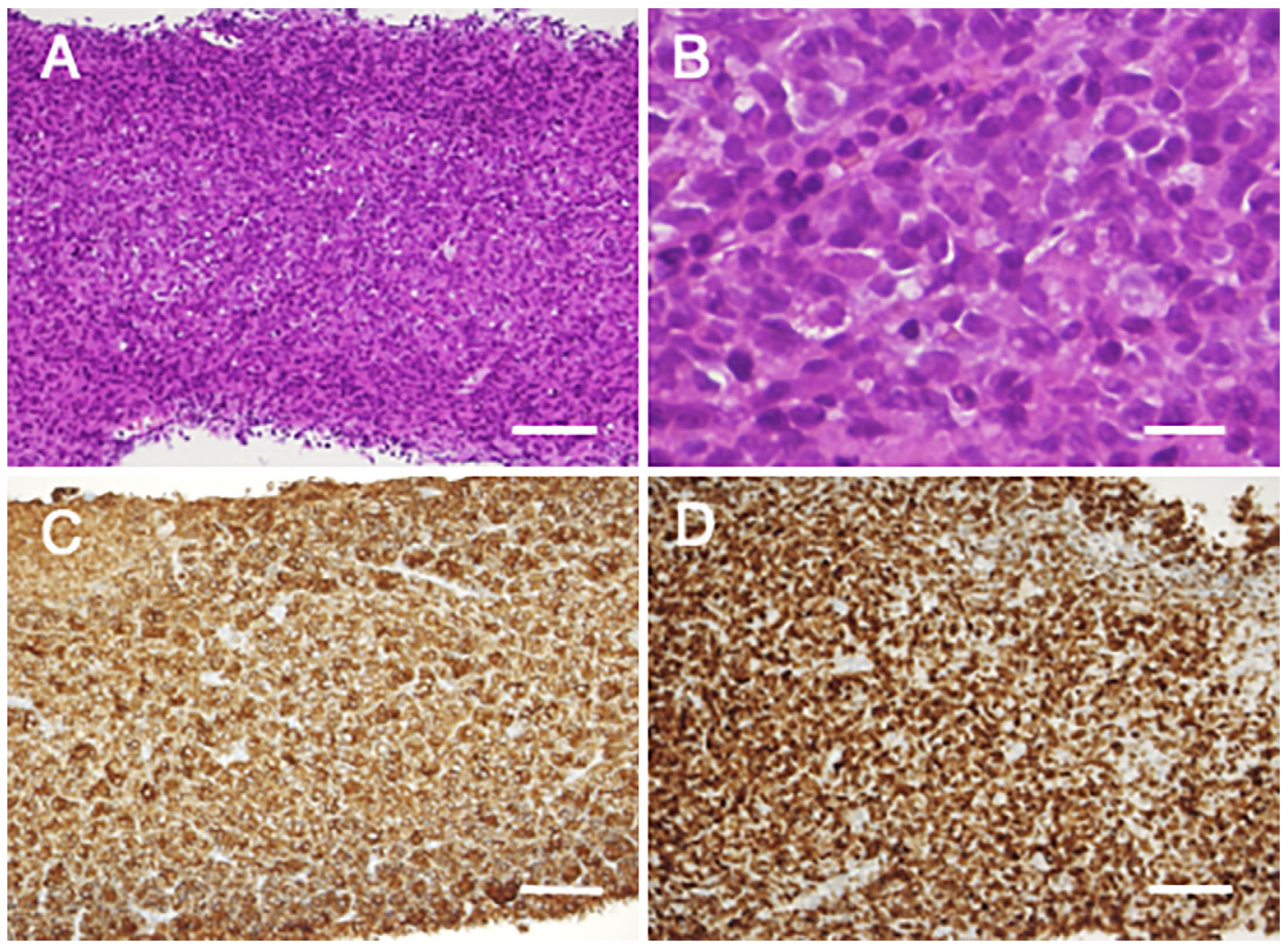

Figure 4. Images of a tumor biopsy specimen. (A) Hematoxylin and Eosin (H\&E) staining, low magnification. (B) H\&E staining, high magnification. (C, D) Immunostaining showed that the cells were positive for B-cell markers, such as $\mathrm{CD20}$ (C) and CD79a (D), and the tumors were diagnosed as diffuse large B-cell lymphoma (DLBCL). Bars indicate $100 \mu \mathrm{m}$ in A, C, D and $20 \mu \mathrm{m}$ in B.

MTX-LPD is generally diagnosed based on both a pathological diagnosis of malignant lymphoma and a history of MTX administration, taking into account EBV positivity.

Thus far, 16 cases with MTX-LPD in the liver have been reported $(10,11,23)$, and the duration of MTX has ranged from 24 to 120 months. Therefore, the MTX duration of 17 years in the present case is the longest yet recorded. Regarding imaging findings, it was reported that liver tumors of MTX-LPD appeared as low-density areas on plain CT images and that most tumors were hypovascular on contrastenhanced CT, although some showed mild ring enhancement (10). Because no typical imaging features of hepatic lymphoma have been reported, it is difficult to distinguish hepatic lymphoma from HCC or metastatic tumors based on imaging findings alone (11). Indeed, the present case showed early enhancement and wash-out in contrastenhanced CT/MRI images, which are typical findings of HCC. Therefore, a pathological diagnosis is essential for MTX-LPD.

No therapy for MTX-LPD has been established, but spontaneous regression after the cessation of MTX was reported in $59 \%$ cases, and the rate of regression was higher in EBVencoded small RNA (EBER)-positive cases than EBERnegative cases (18). If regression is not achieved, intensive chemotherapy is required $(2,8)$. In the present case, we performed TACE before the pathological diagnosis had been made, and TACE was shown to be effective for MTX-LPD, at least in the short term. In addition, MTX cessation three weeks after TACE might have contributed to the regression of LPD in the long term. To our knowledge, there has been only one case report describing the efficacy of TACE for the liver tumors of MTX-LPD, similar to the findings in the present case (24). Because the common point of these cases is the hypervascularity of tumors, TACE may be an option for MTX-LPD in the liver if the tumor is hypervascular and regression is not obtained after MTX discontinuation.

In conclusion, for liver tumors found in patients being treated with MTX, MTX-LPD should be considered as a differential diagnosis, even in patients with chronic liver diseases whose tumors are similar to HCC on imaging tests. Although observation is the first option for MTX-LPD, TACE may be a viable subsequent option in cases with hypervascular liver lesions.

The authors state that they have no Conflict of Interest (COI).

\section{References}

1. Kameda H, Fujii T, Nakajima A, et al. Japan College of Rheumatology guideline for the use of methotrexate in patients with rheumatoid arthritis. Mod Rheumatol 29: 31-40, 2019.

2. Harigai M. Lymphoproliferative disorders in patients with rheumatoid arthritis in the era of widespread use of methotrexate: a review of the literature and current perspective. Mod Rheumatol 28: 
$1-8,2018$.

3. Saito $S$, Takeuchi T. Immune response in LPD during methotrexate administration (MTX-LPD) in rheumatoid arthritis patients. J Clin Exp Hematop 59: 145-155, 2019.

4. Baecklund E, Iliadou A, Askling J, et al. Association of chronic inflammation, not its treatment, with increased lymphoma risk in rheumatoid arthritis. Arthritis Rheum 54: 692-701, 2006.

5. Hashimoto A, Chiba N, Tsuno $\mathrm{H}$, et al. Incidence of malignancy and the risk of lymphoma in Japanese patients with rheumatoid arthritis compared to the general population. J Rheumatol 42: 564$571,2015$.

6. Kamel OW, van de Rijn M, Weiss LM, et al. Brief report: reversible lymphomas associated with Epstein-Barr virus occurring during methotrexate therapy for rheumatoid arthritis and dermatomyositis. N Engl J Med 328: 1317-1321, 1993.

7. Gaulard P, Swerdlow S, Harris N, Jaffe E, Sundstrom C. Other iatrogenic immunodeficiency-associated lymphoproliferative disorders. In: World Health Organization Classification of Tumours of Haematopoietic and Lymphoid Tissues. 4th ed. Swerdlow S, Harris N, Jaffe E, Pileri S, Stein H, Eds. IARC Press, Lyon, 2008: 350-351.

8. Niitsu N, Okamoto M, Nakamine H, Hirano M. Clinicopathologic correlations of diffuse large B-cell lymphoma in rheumatoid arthritis patients treated with methotrexate. Cancer Sci 101: 1309-1313, 2010.

9. Inui $\mathrm{Y}$, Matsuoka H, Yakushijin K, et al. Methotrexate-associated lymphoproliferative disorders: management by watchful waiting and observation of early lymphocyte recovery after methotrexate withdrawal. Leuk Lymphoma 56: 3045-3051, 2015.

10. Mizusawa $T$, Kamimura $K$, Sato $H$, et al. Methotrexate-related lymphoproliferative disorders in the liver: case presentation and mini-review. World J Clin Cases 7: 3553-3561, 2019.

11. Ono R, Kumagae $T$, Uojima $H$, et al. Hepatic methotrexateassociated lymphoproliferative disorders identified by multiple liver tumors: a case report and review of the literature. J Med Case Rep 13: 196, 2019.

12. Ishii M, Gama H, Chida N, et al. Simultaneous measurements of serum alpha-fetoprotein and protein induced by vitamin $\mathrm{K}$ absence for detecting hepatocellular carcinoma. South Tohoku District Study Group. Am J Gastroenterol 95: 1036-1040, 2000.

13. Li C, Zhang Z, Zhang P, Liu J. Diagnostic accuracy of desgamma-carboxy prothrombin versus alpha-fetoprotein for hepatocellular carcinoma: a systematic review. Hepatol Res 44: E11-E25,
2014.

14. Bruix J, Sherman M; American Association for the Study of Liver D. Management of hepatocellular carcinoma: an update. Hepatology 53: 1020-1022, 2011.

15. Kudo M, Izumi N, Kokudo N, et al. Management of hepatocellular carcinoma in Japan: Consensus-Based Clinical Practice Guidelines proposed by the Japan Society of Hepatology (JSH) 2010 updated version. Dig Dis 29: 339-364, 2011.

16. Ellman MH, Hurwitz H, Thomas C, Kozloff M. Lymphoma developing in a patient with rheumatoid arthritis taking low dose weekly methotrexate. J Rheumatol 18: 1741-1743, 1991.

17. Hoshida Y, Xu JX, Fujita S, et al. Lymphoproliferative disorders in rheumatoid arthritis: clinicopathological analysis of 76 cases in relation to methotrexate medication. J Rheumatol 34: 322-331, 2007.

18. Ichikawa A, Arakawa F, Kiyasu J, et al. Methotrexate/iatrogenic lymphoproliferative disorders in rheumatoid arthritis: histology, Epstein-Barr virus, and clonality are important predictors of disease progression and regression. Eur J Haematol 91: 20-28, 2013.

19. Kameda T, Dobashi H, Miyatake N, et al. Association of higher methotrexate dose with lymphoproliferative disease onset in rheumatoid arthritis patients. Arthritis Care Res (Hoboken) 66: 13021309, 2014.

20. Mariette $X$, Cazals-Hatem D, Warszawki J, et al. Lymphomas in rheumatoid arthritis patients treated with methotrexate: a 3-year prospective study in France. Blood 99: 3909-3915, 2002.

21. Salloum E, Cooper DL, Howe G, et al. Spontaneous regression of lymphoproliferative disorders in patients treated with methotrexate for rheumatoid arthritis and other rheumatic diseases. J Clin Oncol 14: 1943-1949, 1996.

22. Yamakawa N, Fujimoto M, Kawabata D, et al. A clinical, pathological, and genetic characterization of methotrexate-associated lymphoproliferative disorders. J Rheumatol 41: 293-299, 2014.

23. Watanabe S, Manabe O, Hirata K, et al. The usefulness of (18)FFDG PET/CT for assessing methotrexate-associated lymphoproliferative disorder (MTX-LPD). BMC Cancer 16: 635, 2016.

24. Watanabe S, Takahashi K, Funakoshi K, Terai S. Methotrexateassociated lymphoproliferative disorder: a rare case of large liver tumors. Hepatology 70: 748-750, 2019.

The Internal Medicine is an Open Access journal distributed under the Creative Commons Attribution-NonCommercial-NoDerivatives 4.0 International License. To view the details of this license, please visit (https://creativecommons.org/licenses/ by-nc-nd/4.0/).

(C) 2020 The Japanese Society of Internal Medicine

Intern Med 59: 2255-2260, 2020 